\title{
Flanched-Cross: An Efficient Cell Geometry for Beam Steering Metasurfaces with Orthogonal Polarisation Handling Capability
}

This paper was downloaded from TechRxiv (https://www.techrxiv.org).

LICENSE

CC BY-NC-SA 4.0

SUBMISSION DATE / POSTED DATE

04-01-2022 / 07-01-2022

\section{CITATION}

Ali, Haider; Esselle, Karu; Mukhopadhyay, Subhas (2022): Flanched-Cross: An Efficient Cell Geometry for Beam Steering Metasurfaces with Orthogonal Polarisation Handling Capability. TechRxiv. Preprint. https://doi.org/10.36227/techrxiv.17868659.v1

$\mathrm{DOI}$

10.36227/techrxiv.17868659.v1 


\title{
Flanched-Cross: An Efficient Cell Geometry for Beam Steering Metasurfaces with Orthogonal Polarisation Handling Capability
}

\author{
Haider Ali*1, Karu P. Esselle ${ }^{2}$ and Subhas Makhupadyay ${ }^{1}$ \\ ${ }^{1}$ School of Engineering, Macquarie University, NSW, Sydney, Australia \\ ${ }^{2}$ School of Electrical \& Data Engineering, University of Technology Sydney, NSW, Australia \\ *haider.ali@hdr.mq.edu.au
}

\begin{abstract}
Geometry plays an important part in the characteristics of meta-cells used to design beam steering metasurfaces. One of the most desirables aspects of these cells is a large phase shift range that can be achieved with good transmission amplitude. However, the existing and most commonly used geometries for these cells are not able to produce a complete $360^{\circ}$ phase range with acceptable level of transmission amplitude. In this article we present a new cell geometry, Flanched-Cross, that has superior transmission properties due to its unique shape and parametric variability than the commonly used geometries. The results are verified in simulation and further confirmed through prototyping and measurement. One- and twodimensional steering are also performed for a dual-polarised base array to confirm the applicability of Flanched-Cross cell for beam steering purposes.
\end{abstract}

Index Terms - Phase shift, Phase range, phase transformation, Transmission Amplitude, Electromagnetic transmission, Metasurface, Meta-cell, Beam steering, Array, Geometry, Antenna, Electromagnetic waves, radiation pattern, microwave radiation, Electromagnetic wave manipulation.

\section{INTRODUCTION}

B eam steering of antennas is an essential task in communication [1] [2], especially, for SatCom-on-themove applications [3]. Beam steering can be achieved either electronically, mechanically, or hybrid techniques where both electronic and mechanical steering are combined for optimal results [4] [5]. Electronic beam steering needs phase shifters and individual feeding to each element of the array which are quite expensive if the antenna terminal is to be used for civilian market. Mechanical steering systems are relatively cheaper but they are bulky and have large dimensions restricting their use for portable communication. Hybrid techniques [6] [7], though, try to minimise these limitations but still not to an extent that such terminals can be used for portable SatCom. Beam steering using passive metasurfaces are gaining popularity recently for applications that demand low profile of the terminal, low cost,

This paragraph of the first footnote will contain the date on which you submitted your paper for review. It will also contain support information, including sponsor and financial support acknowledgment. For example, "This work was supported in part by the U.S. Department of Commerce under Grant BS123456." low power consumption, and ease of portability.

Traditional square patches [8] [9] are usually used, due to their simple geometry, for the design of the meta-cells that are combined to construct a beam steering metasurfaces. However, as it was shown in [10], the traditional square patch geometry does not produce efficient results. The popular three metal layers of square patches are not sufficient to produce the complete $360^{\circ}$ transmission phase range required for beam steering metasurfaces. Moreover, majority of the phases are produced by cells that have large metal area which results into losses, high side lobe levels and other undesirable behaviour. Therefore, it is customary to investigate other geometries for unit cell design to overcome the limitations of the traditional square patch cell. In this article, we will investigate a special geometry for cell design which will be referred to as FlanchedCross or interchangeably FLC throughout this article.

We provide more details about this geometry in section II. Then, we demonstrate the construction of a near-field steering metasurface (NFSS) that will be used for one-dimensional (1D) steering of a dual polarised (DP) microstrip antenna array. Results will be provided both from simulation and measurement. In section IV, two-dimensional (2D) steering is performed using a pair of FLC NFSS for a larger version of DP array followed by the conclusion of our article in section $\mathrm{V}$.

\section{DESIGN OF FlanCHED-CROSS CELL}

Figure 1 shows the design procedure of the Flanched-Cross (FLC) cell in a simulation environment of CST Microwave Studio. First, we start by drawing a square cell with dielectric and copper layers similar to the design of a square patch cell shown in Figure 4. The cell has two layers of dielectric and three layers of copper sandwiched between the dielectric layers. The size of the cell is selected to be $s \times s$, where $s=9 \mathrm{~mm}$. Dielectric RT5880 was chosen for its unique properties required for our design.

After drawing the square patch, we draw four identical

The next few paragraphs should contain the authors' current affiliations, including current address and e-mail. For example, F. A. Author is with the National Institute of Standards and Technology, Boulder, CO 80305 USA (email: author@ boulder.nist.gov). 
circular patches with same radii, $r_{f}$. The centre of the circular patches are the corners of the cell. Then, the circular patches are subtracted from the square patch leaving a Flanched-Cross shape as shown in Figure 1 (a). Figure 1 (b) shows the screenshot of the 3D design of the cell in CST Microwave Studio while Figure 1 (c) shows the top layer of the cell when the value of $r_{f}$ is varied. Notice how the shape of the FlanchedCross changes as the radius $r_{f}$ is increased or decreased. Especially, when $r_{f} \geq s / 2$ the FLC shape reduces to a diamond shape.

It is important to note that the radii of the cut-outs of the top and bottom layers are kept the same and are indicated as $r_{f_{\text {out }}}$. The radii of the cutouts of the middle layer are, however, kept independent of top and bottom layers and are denoted as $r_{f_{i n}}$. For simulation, two Floquet ports were used similar to the one used in [10] and similar boundary conditions were applied here for the unit cell.

\section{A. Properties of Flanched-Cross Cell}

Now that the design of the cell is ready, we can explore its properties in detail. To observe its transmission properties, the dimensions of the cell are varied between maximum and minimum values parametrically. The dimensions that are varied are $r_{f_{\text {in }}}$ and $r_{f_{\text {out }}}$. All other parameters are kept constant. The variation in $r_{f_{\text {in }}}$ and $r_{f_{\text {out }}}$ not only varies the size of the metal area but also changes its shape and that is why a large number of transmission phase shifts can be achieved with FLC cell as opposed to the traditional square patch cell which does not change shape with parametric variation [8].

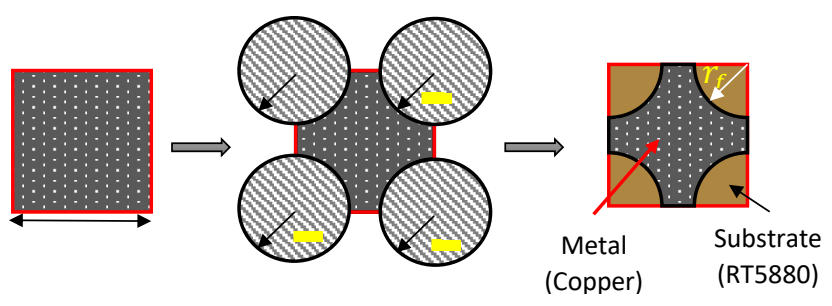

$\begin{array}{ccc}\text { Step 1: } & \text { Step 2: Draw circular } & \text { Step 3: Subtract } \\ \text { Design a } & \text { patches of radius } r_{f} \text { with } & \text { circular patches } \\ \text { Square Cell } & \text { corners of the square as } & \text { from the square } \\ & \text { their centres } & \text { patches }\end{array}$

(a)

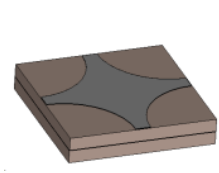

(b)
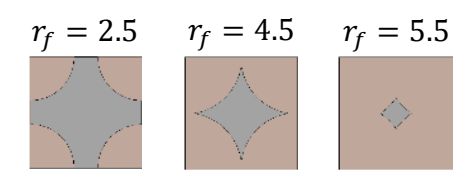

(c)
Figure 1. Flanched-Cross unit cell design: (a) the design process in a $3 D$ EM simulator, (b) a 3D cell designed in CST Microwave Studio, and (c) cell geometry for different radii of the cut-out.

Figure 2 shows the transmission's phase vs amplitude plot when only $r_{f_{\text {out }}}$ is varied while $r_{f_{\text {in }}}$ is kept at a constant value of $5.8 \mathrm{~mm}$. Notice the trend of transmission phase in Figure 2 (a). The phase monotonically increases with $r_{\text {fout }}$. Also, it is to be noted that the maximum value for both $r_{f_{\text {in }}}$ and $r_{f o u t}$ is 6.2 $\mathrm{mm}$ as beyond this value the FLC shape diminishes. For this particular arrangement, transmission's amplitude is greater than $-3 \mathrm{~dB}$ when $r_{f_{\text {out }}}>3.5 \mathrm{~mm}$ while the phase range achievable within the limits of $-3 \mathrm{~dB}$ is about $-30^{\circ}$ to $+150^{\circ}$. However, for other arrangements, both amplitude and phase range vary significantly as will be shown later. The amplitude decreases with the increase in the metal area as we reduce the value of $r_{f_{\text {out }}}$, with a fixed value of $r_{f_{\text {in }}}$, as shown in Figure 2 (c). The larger the metal area the larger the reflection of the incident wave resulting into lower transmission.

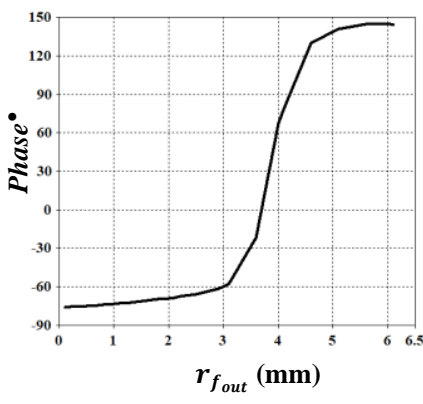

(a)

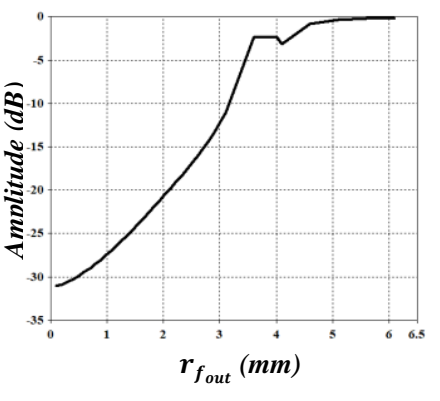

(b)

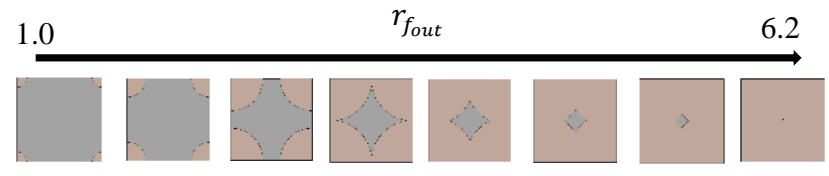

(c)

Figure 2. Transmission properties of FLC for a fixed $\mathrm{r}_{\mathrm{f}_{\mathrm{in}}}=5.8 \mathrm{~mm}$ while $\mathrm{r}_{\mathrm{f}_{\text {out }}}$ was varied from minimum to maximum value: (a) phase and (b) amplitude.

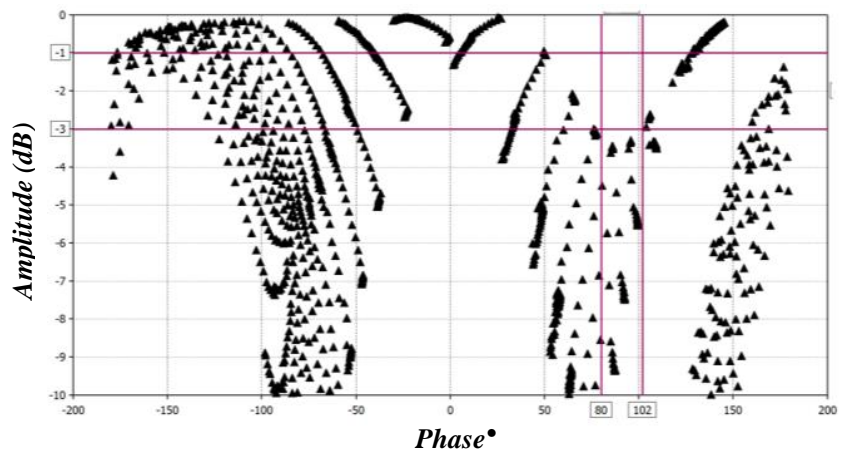

Figure 3. Transmission properties of Flanched-Cross for all parametric variation of $r_{f_{\text {in }}}$ and $r_{f_{\text {out }}}$.

Now that we have a preliminary understanding of the behaviour of the FLC cell we can proceed with its full parametric study. The values of $r_{f_{\text {in }}}$ and $r_{f_{\text {out }}}$ were varied from minimum $(0 \mathrm{~mm})$ to maximum $(6.2 \mathrm{~mm})$ parametrically and the transmission properties were recorded during simulation. A phase vs amplitude plot for all parametric variation is given in Figure 3. The FLC cell is able to produce the whole phase range 
from $-180^{\circ}$ to $+180^{\circ}$ with amplitude greater than $-3 \mathrm{~dB}$ while a substantial part of this range has amplitude greater than $-1 \mathrm{~dB}$. This is quite remarkable when compared to the traditional square patch cell. As was shown in [8], square cell can produce only a phase range of $\approx 300^{\circ}$ with amplitude greater than $-3 \mathrm{~dB}$. The phase range achievable for amplitude greater than $-1 \mathrm{~dB}$ with the square cell is even shorter.

The phase range that can be covered by most commonly used geometries in literature (shown in Figure 4) are given in Table 1. It is evident from Table 1 that FLC outperforms all the cells in terms of phase coverage and amplitude. Specifically, for amplitude greater $-1 \mathrm{~dB}$, which is critical for near-field metasurface design, the phase range covered by FLC is significantly larger than all other geometries.

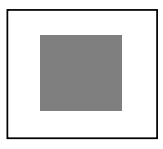

Square Patch [8]

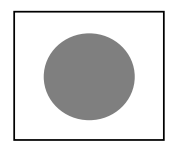

Circular

Patch [11]

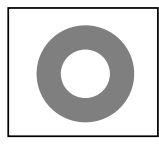

Annular

Ring [12]
Figure 4. Commonly used geometries for cell design. White colour represents substrate while grey represents metal area.

Table 1. Comparison of phase coverage by FLC cell with other commonly used cells. Two layers of dielectric and 3 layers of metal are used for each cell.

\begin{tabular}{|l|c|c|c|c|}
\hline \multirow{2}{*}{ Cell type } & \multicolumn{2}{|c|}{$\begin{array}{c}\text { Phase }^{\circ} \text { range for } \\
\text { Amplitude }>-1 \mathrm{~dB}\end{array}$} & \multicolumn{2}{c|}{$\begin{array}{c}\text { Phase } \\
\text { Amplitude }>-3 \mathrm{~dB}\end{array}$} \\
\cline { 2 - 5 } & Range & $\begin{array}{c}\text { No. of } \\
\text { phases }\end{array}$ & Range & $\begin{array}{c}\text { No. of } \\
\text { phases }\end{array}$ \\
\hline $\begin{array}{l}\text { Square } \\
\text { Patch [8] }\end{array}$ & -30 to 145 & 175 & -155 to 145 & 300 \\
\hline $\begin{array}{l}\text { Circular } \\
\text { Patch [11] }\end{array}$ & $\begin{array}{c}-12 \text { to } 73 \\
\text { and } \\
125 \text { to } 145\end{array}$ & 105 & -12 to 146 & 158 \\
\hline $\begin{array}{l}\text { Annular } \\
\text { Ring [12] }\end{array}$ & $\begin{array}{c}-180 \text { to }-160 \\
\text { and } \\
-24 \text { to } 180\end{array}$ & 224 & $\begin{array}{c}-180 \text { to }-133 \\
\text { and } \\
-24 \text { to } 180\end{array}$ & 251 \\
\hline $\begin{array}{l}\text { Flanched- } \\
\text { Cross (this } \\
\text { work) }\end{array}$ & $\begin{array}{c}-180 \text { to } 50 \\
\text { and } \\
130 \text { to } 180\end{array}$ & 310 & $\begin{array}{c}-180 \text { to } 80 \\
\text { and } \\
102 \text { to } 180\end{array}$ & 338 \\
\hline
\end{tabular}

The bandwidth of a beam steering metasurface is determined by several parameters such as the individual cells' bandwidth, the base antenna or array bandwidth (bandwidth of the source), substrate material and thickness etc. Since the substrate material and thickness has already been selected, we will not investigate their effect on the overall bandwidth of the metasurface.

The transmission properties of each individual cell are different than the others and therefore, their bandwidth will also be different. However, it is useful to look into their behaviour in terms of bandwidth. Figure 5 shows the plot of transmission phase and amplitude over the frequency range 10.5 to $12.5 \mathrm{GHz}$ with the centre frequency being $11.5 \mathrm{GHz}$. The plot represents the behaviour of FLC cell over frequency when one of its parameters i.e., $r_{f_{\text {out }}}$ is varied from minimum to maximum value. The value of $r_{f_{\text {in }}}$ is kept constant $(5.8 \mathrm{~mm})$ to better understand the effect of surface area on the bandwidth. The slope of the phase curve is relatively lower when $r_{f_{\text {out }}}$ is either too low (large metal area) or too high (low metal area) which means that the phase does not change very sharply in these cases. This means the behaviour of the cell will not change too much with frequency and will result into larger bandwidth. However, when the value of $r_{f_{\text {out }}}$ is around the average mean of the range i.e., around $3.5 \mathrm{~mm}$ then the phase variation is high over frequency. Also, as previously noted, the amplitude of the transmission is inversely proportional to the metal area, i.e., the larger the metal area (lower $r_{f_{\text {out }}}$ ) the lower the amplitude and vice versa. It is important to note that large phase variation is desirable in a metasurface design to generate as many phase shifts as possible. However, that comes at the price of limited bandwidth as demonstrated by the results shown in Figure 5.
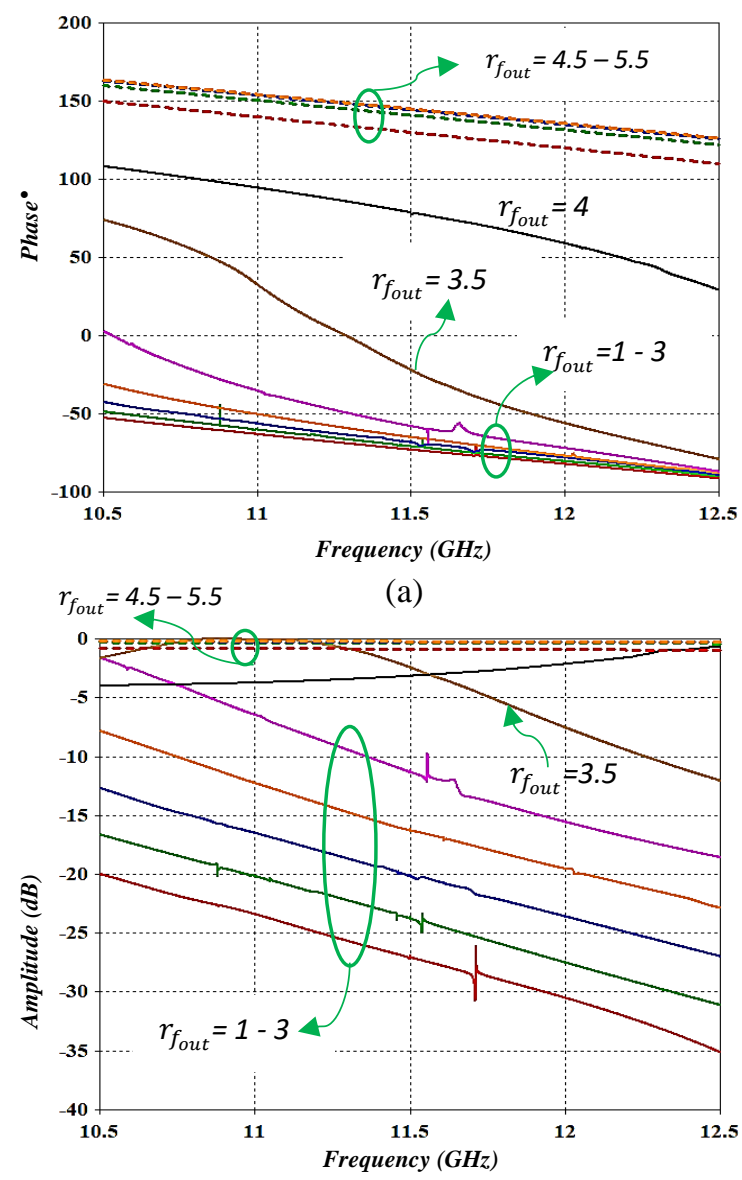

(b)

Figure 5. Bandwidth of Flanched-Cross cell: (a) transmission's phase and $(b)$ amplitude over frequency.

Since each cell has a different bandwidth than others, characterising bandwidth of a metasurface in terms of individual cells is not very useful. Instead, it is more useful to find the bandwidth of the metasurface itself. One way to do that is to simulate the metasurface combined with the base antenna. However, this arrangement will embed the properties of the base antenna or array and the end result will not accurately represent the metasurface under investigation. Therefore, a 
more accurate approach will analyse the metasurface with plane wave input as was assumed during the initial cell design and will be discussed in more detail later on in this article.

Next, we investigate the properties of Flanch-Cross in terms of polarisation handling as we will construct a beam steering metasurface to steer the beam of a dual polarised (DP) array in Section III and IV. The array is radiating with linear horizontal and vertical polarisations as well as circular polarisation. Therefore, the beam steering metasurface should be able to handle linear and circular polarisation. Ideally, the metasurface should produce similar results for vertical and horizontal polarised input waves. Due to its $4^{\text {th }}$ order $\left(90^{\circ}\right)$ rotational symmetry, it is expected that Flanched-Cross metasurface will not alter the polarisation of the incident waves either with linear or circular polarisation. To verify this, the FLC cell was simulated with plane wave input applied from various azimuth angles as shown in Figure 6 (a). The angle $\phi_{\text {in }}$ corresponds to the angle of the E-field vector with respect to $\mathrm{x}$-axis. Both Transverse Electric (TE) and Transverse Magnetic (TM) modes were applied. We simulated the FLC cell with various values of $\phi_{\text {in }}$ and the resulting transmission properties are shown in Figure 6 (b) and (c) where we see that the transmission properties are unaffected by the azimuth scan angle. Since the polarisation of the base array is either linear or circular, the $90^{\circ}$ rotational symmetry of the FLC cell will adequately handle the polarisation without affecting it significantly.
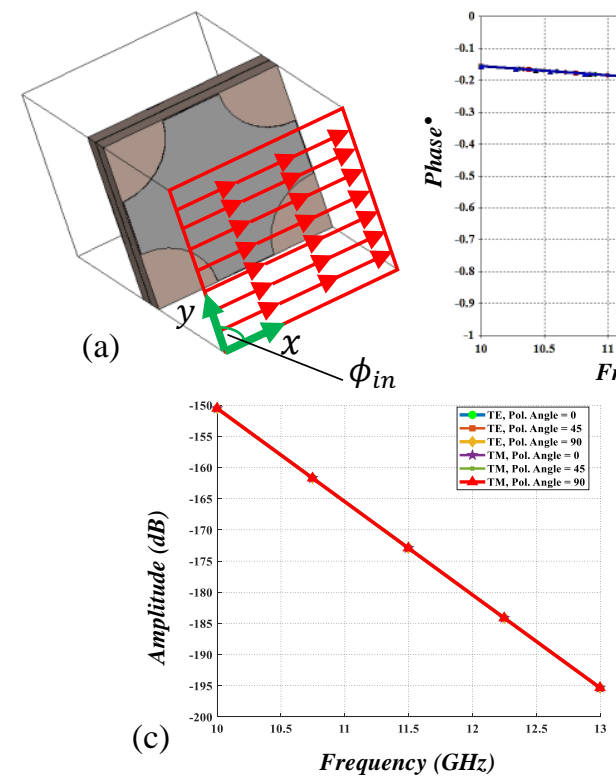

Figure 6. Cell's polarisation check: (a) E-field vector orientation, (b) cell's phase and (c) amplitude behaviour with changing polarisation.

\section{COnStruction OF A SteEring Metasurface Using FLC CELL}

We design a beam steering metasurface in this section that will steer the wave passing through it to an elevation angle $\theta=29^{\circ}$. We will need a number of cells having different phase shifts $\left(\phi_{e}\right)$ to construct a beam steering metasurface. These phase shifts are calculated using classical beamforming techniques used for linear antenna arrays, i.e., $\phi_{e}=\frac{2 \pi}{\lambda} d \sin \theta$, where $\lambda$ is the operating wavelength, $d$ is the distance between two consecutive elements which is assumed to be the same for all elements, and $\theta$ is the desired elevation angle towards which the beam of the array will be steered. For $\theta=29^{\circ}$ the value of $\phi_{e}=60^{\circ}$.

In the case of NFSS, a single column of cells corresponds to a single element in a linear array as shown in Figure 8. So, each column should produce a phase difference equal to $\phi_{e}$ in the incoming wave. This corresponds to a $60^{\circ}$ discretisation required to design such a metasurface which means there will be six distinct phase shifts within a complete $360^{\circ}$ cycle, i.e., $\phi_{e}=0^{\circ}, 60^{\circ}, 120^{\circ}, 180^{\circ}, 240^{\circ}$, and $300^{\circ}$. Note that the specific value of starting phase is not fixed and can be chosen randomly as long as the subsequent phases are selected continuously with the selected discrete step within a complete $360^{\circ}$ cycle. For example, we can select the first phase value to be $-180^{\circ}$ and then the subsequent phases will be: $-120^{\circ},-60^{\circ}, 0^{\circ}, 60^{\circ}, 120^{\circ}$, and $180^{\circ}$. Then, for each phase shift a corresponding FLC is designed that has the same transmission phase as $\phi_{e}$. The dimensions of the FLC cells corresponding to these phase shifts are given in Table 2. Here we have chosen the phase range of the metasurface to be from $-180^{\circ}$ to $180^{\circ}$ incorporating complete $360^{\circ}$ phase range. Notice that $180^{\circ}$ and $-180^{\circ}$ corresponding to the same phase so only one value will be considered for inclusion in the metasurface design.

Table 2. Dimensional values of the Flanched-Cross cells to construct a metasurface for $\theta=29^{\circ}$.

\begin{tabular}{ccccc}
\hline \hline $\begin{array}{c}\text { Required } \\
\phi_{e}{ }^{\circ}\end{array}$ & $\begin{array}{c}r_{f_{\text {in }}} \\
(\mathrm{mm})\end{array}$ & $\begin{array}{c}r_{f_{\text {out }}} \\
(\mathrm{mm})\end{array}$ & $\begin{array}{c}\text { Cell's } \\
\text { actual } \\
\text { phase }^{\circ}\end{array}$ & $\begin{array}{c}\text { Cell's } \\
\text { amplitude }(\mathrm{dB})\end{array}$ \\
\hline-180 & 2.3 & 3.0 & -177.2 & -1.06 \\
-120 & 2.7 & 2.4 & -119.5 & -0.95 \\
-60 & 1.5 & 3.5 & -60.3 & -1.10 \\
0 & 4.7 & 3.7 & -0.3 & -0.58 \\
60 & 4.3 & 4.0 & 60.0 & -1.01 \\
120 & 4.6 & 4.5 & 120.9 & -0.86 \\
\hline
\end{tabular}

The final metasurface designed after combining all cells in order is shown in Figure 8. The metasurface was extended in size in all directions by duplicating appropriate cells to cover the surface area of a dual-polarised (DP) base array shown in Figure 11. In the next section, we will present the steered radiation results when the metasurface is placed on top of DP base array.

\section{A. Applying Flanched-Cross NFSS to a Dual Polarised Array} For demonstration of FLC cell's handling capability of orthogonal polarisations we will use the designed metasurface to steer the beam of a dual polarised (DP) array shown in Figure 11. The array has two sub-arrays both with a Taylor-tapered feed network [13]: vertical and horizontal sub-arrays. Vertical sub-array radiates with vertical linear polarisation while the horizontal sub-array radiates with horizontal linear polarisation. Then, the Flanched-Cross metasurface is placed on top of the 
DP array with a gap of $\lambda_{0} / 2$, the operating wavelength at 11.5 $\mathrm{GHz}$, between them. This gap was further optimised for improved performance.

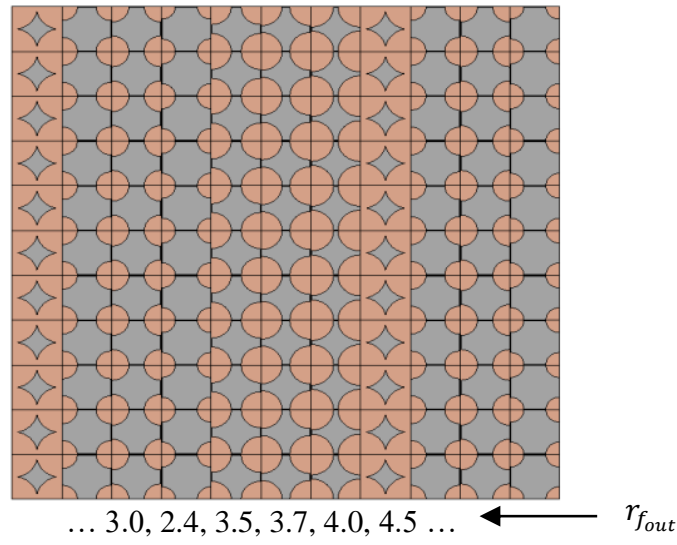

Figure 8. A metasurface made from Flanched-Cross cells for $\theta=$ $29^{\circ}$. Pink represents substrate while grey represents copper.
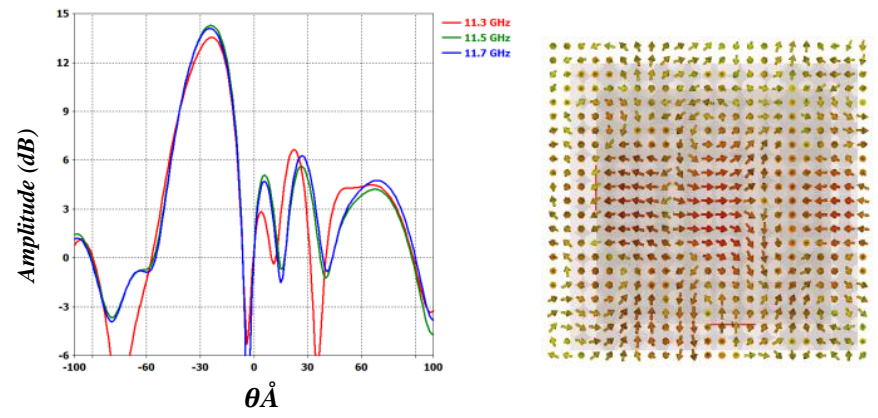

(a)

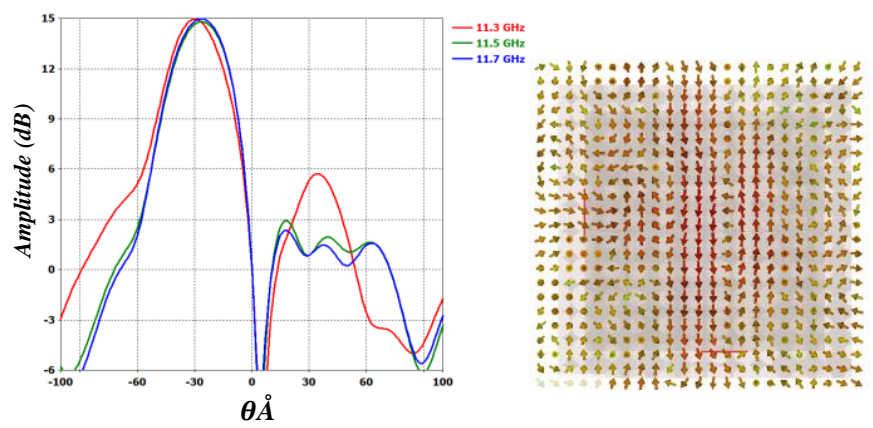

(b)

Figure 9. 1-D beam steering results. (a) horizontal subarray and (b) vertical subarray. Directivity patterns of radiated field are on the left side while a snapshot of E-field vectors showing the polarisation of the E-field on the right side.

The DP array is first operated in horizontal polarisation mode which is activated by exciting only the horizontal sub-array while the vertical sub-array is kept inactive. The FlanchedCross metasurface is placed on top of the DP array such that the phase gradient of the metasurface is aligned with the polarisation of the intended subarray, e.g., along $\mathrm{x}$-axis for horizontal subarray representing the azimuth angle $\phi=0^{\circ}$. The complete structure was simulated in CST Microwave Studio.
The radiation patterns of the steered beam are shown in Figure 9 (a). The beam was successfully steered to $28.3^{\circ}$ with an error of only $0.7^{\circ}$. Moreover, the results are very consistent for various frequencies within $11-12 \mathrm{GHz}$ band as is evident in Figure 9 (a).

The same procedure was carried out for vertical subarray. The radiation patterns of the steered beam are shown in Figure 9 (b). Here, steering was achieved with even less error $\left(0.2^{\circ}\right.$ only). Also, beam patterns are very stable within the frequency range of $11-12 \mathrm{GHz}$. The snapshots of E-field vectors of the steered beam shown in Figure 9 (a) and (b) indicate that both linear polarisations were accurately reproduced without changing their sense or angle verifying the polarisation handling capability of the FLC metasurface.

The Flanched-Cross metasurface was then rotated by several angles $\left(\alpha=90^{\circ}, 120^{\circ}, 270^{\circ}\right)$ around its axis for beam steering in other azimuth directions. This was done when the array was operated in both vertical and horizontal polarisations respectively. Figure 10 shows radiation patterns for the selected values of $\alpha$ for both cases. The steering was done sufficiently accurately within error of $1.7^{\circ}$ in all cases.

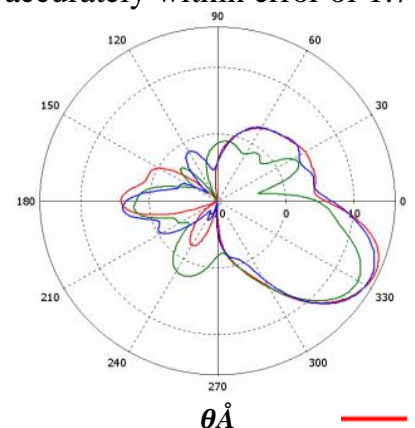

(a)

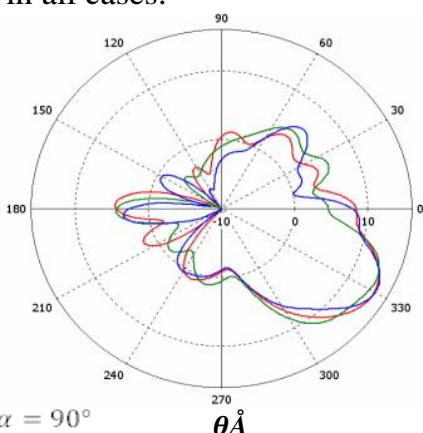

(b)
Figure 10. Patterns of the steered beam for various values of $\alpha:(a)$ horizontal subarray and (b) vertical subarray.

\section{B. Fabrication and Measurement}

Both the base array and FLC NFSS was fabricated and measured in an anechoic chamber for steering. Figure 11 shows the fabricated prototypes. We tested the setup for the two orthogonal polarisations by placing NFSS first at $0^{\circ}$ azimuth and then rotating it to $90^{\circ}$ azimuth. That way the axis of NFSS will be aligned first to horizontal polarisation and then vertical polarisation respectively. In both cases the beam will be steered toward the designed $\theta$ but the main beam will be along the azimuth angle corresponding to the NFSS rotated axis.

The prototypes were measured in anechoic chamber with the probe WR-75, model ANT-WGP-10-15 from NSI-MI Technologies. The measured radiation patterns are shown in Figure 12 where both the co-pol and cross-pol components are plotted. The cross-pol components are quite apart from the copol by at least $-30 \mathrm{~dB}$ in the desired elevation direction. Moreover, the steering results are within $1^{\circ}$ error. However, a slightly higher sidelobe level was observed for vertical subarray than horizontal subarray. 


\section{Full SCALE SteERING OF THE ARRAY’s BEAM}

The steering metasurface presented in previous section can only steer the beam of the base array along one elevation angle, $\theta$, though the beam can be steered towards any azimuth angle by simply rotating the surface. However, this will only allow the beam to rotate along the contour of a cone with an apex angle $\theta$.

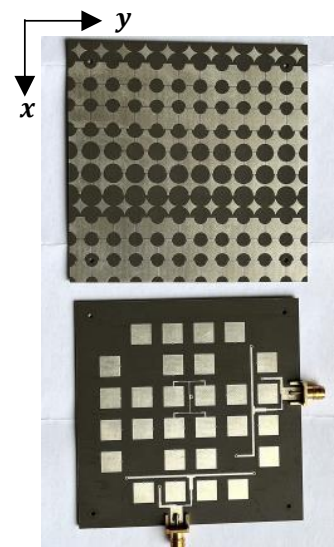

(a)

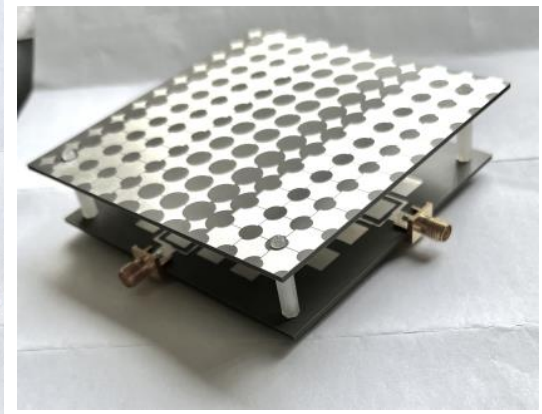

(b)
Figure 11. Fabricated prototypes of the array and FLC NFSS.

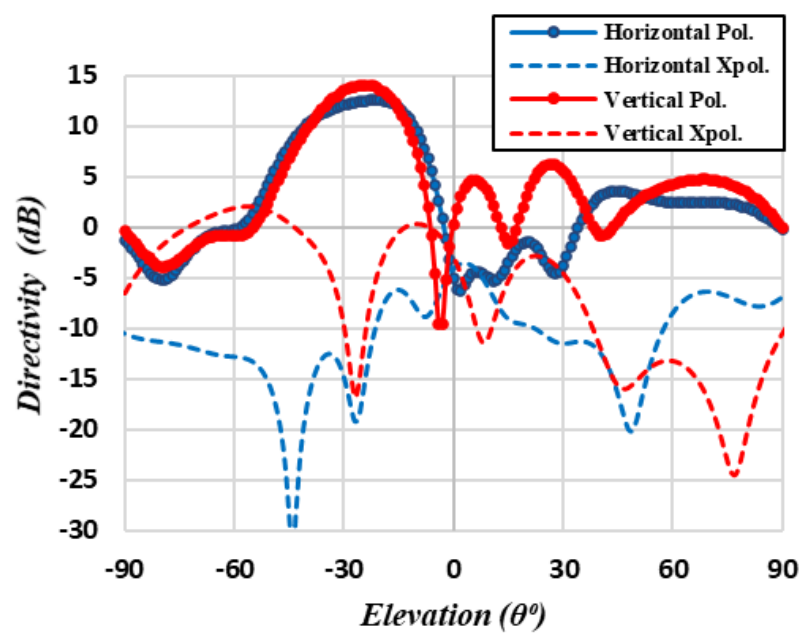

Figure 12. Measured radiation patterns of horizontal and vertical subarrays with their co-pol and cross-pol components.

To steer its beam in any direction in three-dimensional space, we need a different mechanism. The predominantly used mechanism in literature for full-scale steering is to use a pair of steering metasurfaces [8]. The two metasurface system has been modelled mathematically with several different approaches in literature. The two mostly used models are paraxial approximation [14] and a recently reported Phase Method [15]. Due to its more accurate estimation, however, we will only use the Phase Method in this article. Without going into the specifics of the model (which can be consulted if needed in these references [8] [14] [15]) we will simply present the equations that we will be using in this report.

Figure 13 shows the setup for full-scale beam steering which consists of a base array and two NFSS. First NFSS tilts the beam by $\delta_{1}$ degrees in elevation and then the second NFSS tilts it by $\delta_{2}$ degrees. The combined effect of two-times tilting will steer the beam towards a particular direction in spherical coordinates $(\theta, \phi)$.

The elevation and azimuth angles $(\theta, \phi)$ of the steered beam after passing through the pair of NFSS's can be estimated using the phase method [19] and are given by:

$$
\begin{gathered}
\theta=\sin ^{-1}\left(\frac{\sqrt{p_{1}^{2}+p_{2}^{2}+2 p_{1} p_{2} \cos \left(\psi_{1}-\psi_{2}\right)}}{k}\right) \\
\phi=\tan ^{-1}\left(\frac{p_{1} \sin \psi_{1}+p_{2} \sin \psi_{2}}{p_{1} \cos \psi_{1}+p_{2} \cos \psi_{2}}\right)
\end{gathered}
$$

where $p_{1}, p_{2}$ (expressed in $\mathrm{rad} / \mathrm{m}$ ) are the surface phase gradient of NFSS 1 and 2 respectively.

The phase method also provides rough estimates of the rotation angles $\psi_{1}, \psi_{2}$ of NFSS- 1 and 2 required to steer the beam towards a particular $(\theta, \phi)$ as follows:

$$
\psi_{1}=\phi+\frac{1}{2} \cos ^{-1}\left[\frac{(k \sin \theta)^{2}}{2 p^{2}}-1\right]
$$

$$
\psi_{2}=\phi-\frac{1}{2} \cos ^{-1}\left[\frac{(k \sin \theta)^{2}}{2 p^{2}}-1\right]
$$

with $k$ being the wavenumber $(k=2 \pi / \lambda)$.

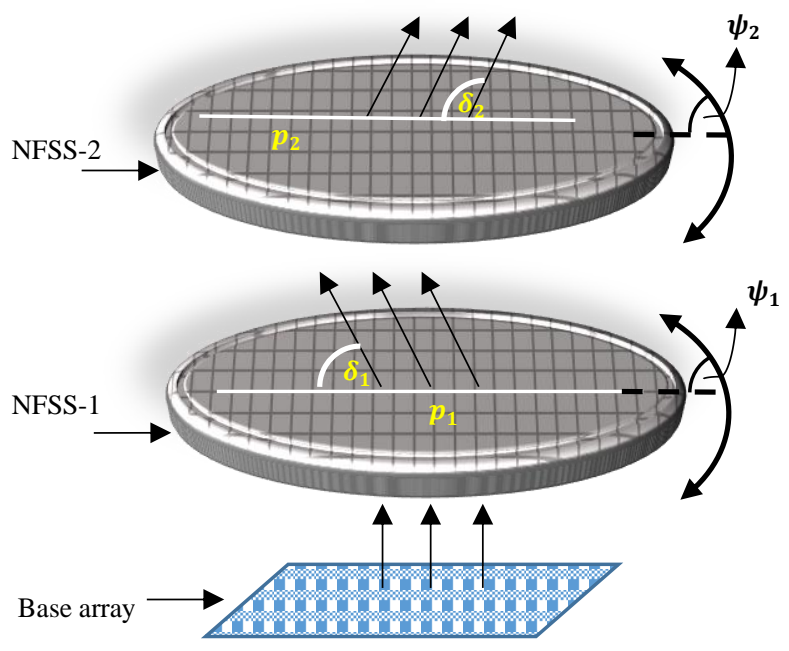

Figure 13. Two-dimensional beam steering using a pair of NFSS's.

We start with the rotational positions of both metasurfaces with these initial values and then optimise according to the results obtained in simulation.

To simplify design, we assume that both NFSS-1 \& 2 have the same phase gradient $p$. The relative rotation of NFSS- $1 \&$ 2 will determine the final direction of the steered beam. When both NFSS's are at their reference point $\left(\psi_{1}=\psi_{2}=0^{\circ}\right)$ then according to (1) \& (2) the beam will point towards maximum elevation angle equal to $2 p / k$ while the azimuth angle of the steered beam will be zero. However, as we keep rotating NFSS- 
2 up to $\psi_{2}= \pm 180^{\circ}$ and keep NFSS- 1 stationary, the beam steers away from previous position towards broadside $(\theta=0)$ The azimuth angle of the steered beam, at any time, will be equal to half the rotation angle of NFSS-2 i.e., $\phi=\psi_{2} / 2$. To rotate the beam towards any desired azimuth angle without changing elevation, we can co-rotate both NFSS's to achieve that. For keeping the beam in one particular azimuth direction while steering it to a desired elevation will require both NFSS's to be rotated counter clockwise with equal rate of rotation.

For 2D steering we will consider a larger version of the base array in simulation for larger directivity and gain value as smallto-medium gain antennas are not suitable for metasurface based 2D beam steering due to the resulting large sidelobe levels. Here, in this case, we are considering a 20×20 size array. The steering is performed for few directions. The steps required for NFSS's rotation and the specific angles of rotation needed to achieve steering in the selected directions are given in Table 3 which were calculated using (1) - (4). First, we rotate only NFSS-2 while NFSS-1 is fixed at its reference position. This will steer the beam to a desired elevation angle. Then, to steer the beam to a particular azimuth direction while keeping it along the same elevation angle, we co-rotate both NFSS's.

After simulation the complete structure of base array and two NFSS with the required rotations, we obtained the radiation patterns of the output EM wave in the main direction of the beam. These results are plotted in Figure 14 where we see the beam was steered towards the selected directions with minimal error $\left(2.4^{\circ}\right)$. Though, it can be observed that the peak directivity value slightly reduces as we steered the beam towards higher elevation angles. Moreover, the sidelobe level is comparative higher for larger elevation angles $\left(\approx-17 \mathrm{~dB}\right.$ for $\left.\theta=35^{\circ}\right)$ than the lower ones $\left(\approx-21 \mathrm{~dB}\right.$ for $\left.\theta=0^{\circ}\right)$.

Table 3. Rotations required to achieve steering in the given directions.

\begin{tabular}{cccc}
\hline \hline $\begin{array}{c}\text { Azimuth } \\
\left(\phi^{\circ}\right)\end{array}$ & $\begin{array}{c}\text { Elevation } \\
\left(\theta^{\circ}\right)\end{array}$ & $\begin{array}{c}1^{\text {st }} \text { rotation: } \\
\text { NFSS-2 only } \\
\left(\psi_{2}^{\circ}\right)\end{array}$ & $\begin{array}{c}2^{\text {nd }} \text { rotation: } \\
\text { Co-rotation of } \\
\text { NFSS-1 \& 2 } \\
\left(\psi_{1}=\psi_{2}^{\circ}\right)\end{array}$ \\
\hline 0 & 10 & 159 & 100 \\
30 & 25 & 128 & 146 \\
70 & 35 & 107 & 196.5 \\
\hline
\end{tabular}

\section{CONCLUSION}

A new cell geometry used for the construction of beam steering metasurfaces and termed as Flanched-Cross was presented in this article. The existing cell designs in literature would require three or even four layers of dielectric to generate complete $360^{\circ}$ phase shifts needed to design a beam steering metasurface. However, due to its unique shape, and specially, the changes in its shape with parametric variation, the Flanched-Cross cell is able to produce the complete $360^{\circ}$ phase shifts with only two layers of dielectric within the transmission amplitude acceptable for metasurface based beam steering. The results were confirmed in simulation and further verified through prototyping and measurement. Furthermore, one- and twodimensional steering was performed for a dual polarised array which successfully demonstrated the orthogonal polarisation handling capability of the Flanched-Cross cell. The steering results were within the error range of only $0.7^{\circ}$ and $2.4^{\circ}$ for $1 \mathrm{D}$ and $2 \mathrm{D}$ steering respectively.

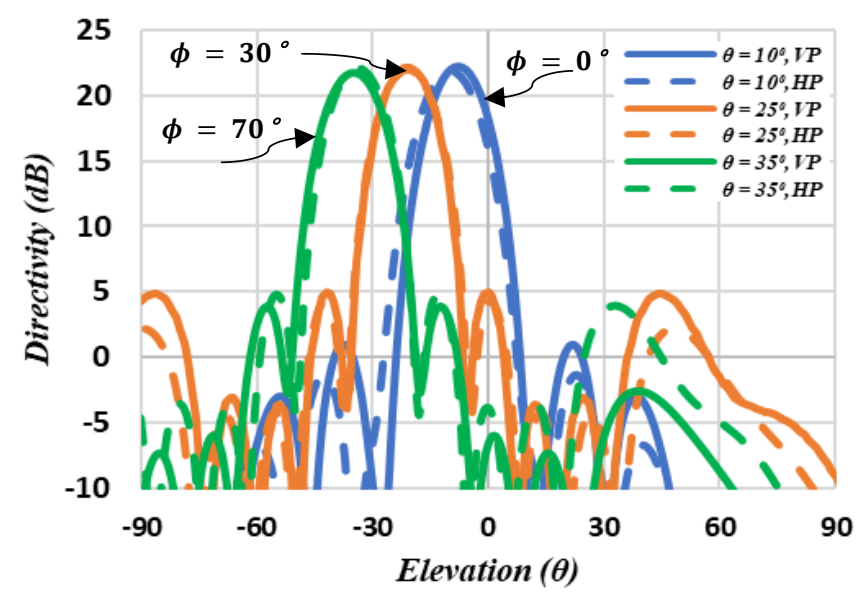

Figure 14. Steering results for the directions given in Table 3.

\section{REFERENCES}

[1] C. A. Balanis, Antenna Theory: Analysis and Design, Wiley, 2016.

[2] K. W. Linnes, W. D. Merrick and R. Stevens, "Ground Antenna for Space Communication System," IRE Transactions on Space Electronics and Telemetry, Vols. SET-6, no. 1, pp. 45-54, 1960.

[3] S. Borisov and A. Shishlov, "Antennas for Satcom-onthe-Move, Review," in 2014 International Conference on Engineering and Telecommunication, Moscow, 2015.

[4] K. P. Esselle, "A Brief Overview of Antenna Technologies for Communications-On-The-Move Satellite Communication Mobile Terminals," in IEEE Antennas and Propagation Society International Symposium, Monteal, 2020.

[5] I. Uchendu and J. Kelly, "Survey of Beam Steering Techniques Available for Millimeter Wave Applications," Progress In Electromagnetics Research $B$, vol. 68, p. 35-54, 2016.

[6] Y. Jung, S. Eom, S. Jeon, A. V. Shishlov and C. Kim, "Novel hybrid antenna design having a shaped reflector for mobile satellite communication applications," in IEEE Antennas and Propagation Society International Symposium, Toronto, 2010.

[7] Y. Jung, A. V. Shishlov and S. Park, "Cassegrain Antenna With Hybrid Beam Steering Scheme for Mobile Satellite Communications," IEEE Transactions on Antennas and Propagation, vol. 57, no. 5, pp. 13671372, 2009.

[8] N. Gagnon and A. Petosa, "Using Rotatable Planar Phase Shifting Surfaces to Steer a High-Gain Beam," 
IEEE Transactions on Antennas and Propagation, vol. 61, no. 6, pp. 3086-3092, 2013.

[9] M. U. Afzal and K. P. Esselle, "Steering the Beam of Medium-to-High Gain Antennas Using Near-Field Phase Transformation," IEEE Transactions on Antennas and Propagation, vol. 65, no. 4, pp. 1680-1690, 2017.

[10] H. Ali, M. U. Afzal, K. P. Esselle and R. M. Hashmi, "Intergration of Geometrically Different Elements to Design Thin Near-Field Metasurfaces," IEEE Access, vol. 8, pp. 225336-225346, 2020.

[11] K. Singh, M. U. Afzal, M. Kovaleva and K. P. Esselle, "Controlling the Most Significant Grating Lobes in Two-Dimensional Beam-Steering Systems With PhaseGradient Metasurfaces," IEEE Transactions on Antennas and Propagation, vol. 68, no. 3, pp. 13891401, 2020.

[12] H. Ali, M. U. Afzal, K. Esselle and R. Hashmi, "Investigating Stacked-Ring Based Cells for Phase Shifting Surfaces," in 2020 IEEE International Symposium on Antennas and Propagation, Montreal, 2020.

[13] T. Taylor, ““”Design of Line-Source Antennas for Narrow Beamwidth and Low Side Lobes," Transactions of the IRE Professional Group on Antennas and Propagation, vol. 3, no. 1, pp. 16-28, 1955.

[14] C. D. McEwen and M. R. Khan, "Beam Steering Method with Improved Sidelobe Response using Dielectric Wedges for Satellite TV Reception," in 14th European Microwave Conference, 1984.

[15] J. Wang and Y. Rahmat-Samii, "Phase Method: A More Precise Beam Steering Model for Phase-Delay Metasurface Based Risley Antenna," in 2019 URSI International Symposium on Electromagnetic Theory (EMTS), 2019. 